\title{
Powerful combination therapies
}

\author{
Cancer therapies that target multiple biological pathways take advantage of synergistic killing, and decrease the \\ risk of relapse.
}

1 esearch into the biological mechanisms of cancer progression and the development of effective antitumour therapies are generating a stream of new data and technological possibilities that are pushing forward our knowledge of human biology and spawning strategies to tackle a wide range of cancer types. Although therapeutic modalities that target unrelated biological pathways are developed largely independently, the case for taking advantage of convergent and interdependent biological processes in combination is clear: attacking tumour cells from different angles increases the chance of success, especially given the inherent ability of tumours to adapt to monotherapies, which often leads to relapse from an undetectable state back to the full-blown, often metastatic, disease.

Combining different cancer therapies such as chemotherapy, radiotherapy, targeted therapy, cell therapy and, most recently, immunotherapy - is of course not a new idea. In the clinic, treatment regimens that involve cytotoxic agents and radiotherapy have long been integrated to enhance the efficacy of single-modality treatments; this has led, for some cancers, to lifechanging outcomes. And the combination of chemotherapy and radiotherapy with therapies targeting distinct molecular markers in tumours (for example, specific hormone receptors) has further increased remission rates in some patients.

Some of the more traditional regimens are now being combined with cancer immunotherapy into multipronged therapeutic schemes. As numerous clinical trials explore the potential advantages of combining immunotherapies with established radiotherapy and chemotherapy protocols, clinical oncologists are gaining new insight into which treatment modalities to integrate in order to offer the best results to individual patients: for example, radiotherapy and chemotherapy regimens can be supplemented by strategies that relieve tumour-reactive $\mathrm{T}$ cells from immunosuppressive signals through the systemic administration of monoclonal antibodies that shut down immune checkpoints, by infusing $\mathrm{T}$ cells engineered to recognize and kill cancer cells, or by vaccinating patients to jump-start tumourspecific immune responses. Positive results featuring these combinatorial approaches are

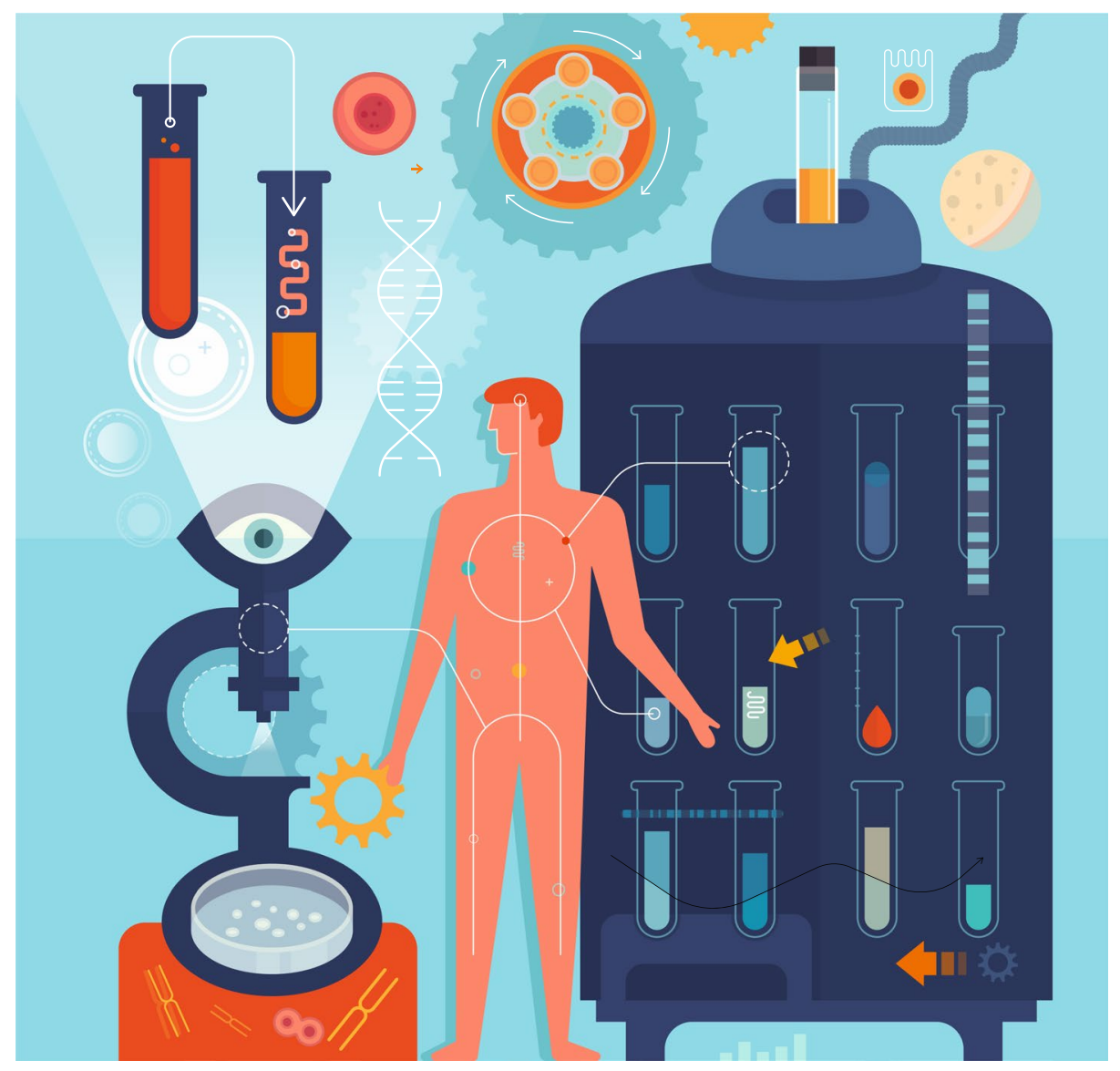

Credit: DrAfter123 / DigitalVision Vectors / Getty Images

prompting the design of multicomponent therapeutics that target the immune system as well as specific immune cells in the tumour microenvironment.

Although effector T cells have been the main target of immunotherapies to date, other immune cells present in the tumour microenvironment are arguably just as amenable to therapeutic intervention. Macrophages (a type of innate immune cell) are often found within solid tumours. Depending on their phagocytic activity, cytokine production and antigen presentation, tumour-associated macrophages (TAMs) can attack tumour cells (M1 subtype) or be largely tumourpermissive (M2 subtype). Targeting and manipulating TAMs is therefore a rational therapeutic approach that can be combined with other therapeutic regimens. Ralph Weissleder and colleagues report in this issue the use of cyclodextrin nanoparticles to deliver R848, a toll-like receptor ligand, to promote the conversion of M2 macrophages into the M1 phenotype as an adjunct modality to checkpoint blockade immunotherapy. R848 inhibited tumour growth in multiple cancer mouse models, an effect that was linked to the M2-to-M1 repolarization of the TAMs. Furthermore, R848 enhanced the efficacy of anti-PD-1 (programmed cell death protein 1) checkpoint blockade in the treatment of an anti-PD-1-resistant tumour.

In a complementary study, Ashish Kulkarni, Shiladitya Sengupta and colleagues describe a self-assembled supramolecule, about $100 \mathrm{~nm}$ in diameter, designed to 
deliver an inhibitor of colony stimulating factor 1 receptor (CSF-1R) and an antibody blocking signal regulatory protein $\alpha$ (SIRP- $\alpha)$. These two inhibitory agents interfere with tumour-permissive processes in TAMs: the activation of CSF-1R that induces the M2 phenotype, and the expression of SIRP- $\alpha$ by cancer cells that activates the inhibitory receptor CD47 (which provides a 'do not eat me' signal) in macrophages. By blocking these two distinct pathways, the supramolecule led to an increase in M1 macrophages in the tumour and to enhanced phagocytic activity, which caused a significant slowdown of tumour growth in multiple tumour models in mice. Overall, both studies demonstrate that the use of nanoparticles for the delivery of therapeutics to TAMs benefits from the phagocytic activity that is characteristic of macrophages. As Heather Gustafson and Suzie Pun discuss in an associated News \& Views, one can "take advantage of the particle-engulfing properties of macrophages to develop nanoparticles that are internalized by TAMs for the delivery of immune-modulating drugs that promote anticancer activity."

Among the established cancer therapies that have been combined with immunotherapy, the integration of radiotherapy with other therapeutic modalities is less developed. One bottleneck is that, although radiation therapy induces DNA damage in cells, the hypoxic microenvironment of solid tumours dampens the oxygen-radical-mediated cytotoxic mechanisms associated with radiotherapy, reducing its efficacy. Kai Yang, Zhuang Liu and colleagues report a radiotherapy-based approach that modulates several tumourmicroenvironment-associated biological processes, including hypoxia, immune activation and immune checkpoint blockade. By injecting into the tumour a hydrogel containing ${ }^{131} \mathrm{I}$-labelled catalase (an enzyme that reduces hydrogen peroxide into water and molecular oxygen), the researchers created a potent anti-hypoxic radiotherapy modality. The hydrogel, which confined the therapeutics to the tumour site, was also combined with CpG (another toll-like receptor ligand) to stimulate local immune cells or with the systemic administration of anti-CTLA-4 (cytotoxic T-lymphocyte-associated protein 4) antibodies for checkpoint blockade. Because radiotherapy-induced tumour-cell death is an immunogenic event that releases tumourassociated antigens, it bolsters de novo immune responses to tumour antigens.

Although the immunogenic events arising from radiotherapy or chemotherapy can be successfully leveraged to support antitumour immunity, cancer vaccines offer more control over the specificity of the antitumour immune response, and may limit off-tumour toxicity resulting from on-target activity. In a Comment, Ugur Sahin and colleagues lay the case for eliciting immune responses to cancer neoantigens - antigens that arise from tumour-specific mutations and that are not found in healthy tissues - as a personalized and highly effective antitumour therapy. Identifying neoantigens and creating highly targeted tumour vaccines that are patientspecific requires a sophisticated and highly coordinated manufacturing set-up, yet it has already yielded promising results in patients with melanoma. And, as with other therapeutic modalities, the precise targeting of tumour vaccines can be combined with the potentiating effects of immune checkpoint inhibitors to yield both effective and targeted therapeutic regimens.

Cancer is a multifactorial, resilient and extremely heterogeneous disease that despite intense research and specific successes has overall remained refractory to therapy. The promises and achievements of combination approaches suggest that personalized, multifaceted therapies could be made effective and broadly applicable. Still, determining and implementing the right combination of therapeutic approaches for each patient will be a huge but unavoidable challenge.

Published online: 9 August 2018 https://doi.org/10.1038/s41551-018-0283-1 